\title{
MODELO PERSONALIZADO \\ DE CALIDAD PARA \\ UN CENTRO EDUCATIVO
}

Isabel Parés Gutiérrez

"Podemos encontrar escuelas de baja calidad dirigidas por buenos directores, pero resultaría muy difícil que un centro de alta calidad no estuviera lidereado por un buen profesional".

Estruch

\section{RESUMEN}

En la actualidad, existe una amplia variedad de modelos de certificación de calidad enfocados a distintos ámbitos, sobre todo orientados al ámbito productivo; también es posible hallarlos para el sector educativo. Sin embargo, la gran mayoría se basan en los primeros y un número menor han sido ideados específicamente para centros escolares, basándose en una evaluación estandarizada y cuantitativa.

Ante esta perspectiva, se propone un modelo de certificación de la calidad educativa que, con base en una norma de calidad, derive en criterios estandarizados y criterios personalizados -que van de lo genérico a lo específico-, con indicadores cualitativos y cuantitativos para ambos casos. Los criterios estandarizados son aquéllos aplicables a toda institución educativa; los personalizados hacen referencia a cómo la filosofía organizacional baña los ámbitos del centro escolar y permite incluir proyectos propios de la institución certificada. 


\section{INTRODUCCIÓN}

En una primera aproximación, este escrito busca apoyar los procesos de mejora en los centros educativos, aclarando algunos conceptos que resultan indispensables para alcanzar niveles de excelencia educativa.

Hoy día se viven, a cada minuto, procesos de apertura e intercambio fomentados por la globalización que ha abierto las fronteras, no sólo de productos y servicios, sino también de información. Este fenómeno mundial expone, a cualquier organización, a ser evaluada; mostrar sus niveles de eficacia y eficiencia; informar cómo lleva a cabo sus procesos y qué resultados obtiene.

Las escuelas no están fuera de este fenómeno. Se han internacionalizado cada vez más, percibiendo la necesidad de implementar nuevos modelos de enseñanza, y se les han exigido mejores resultados en el aprendizaje de los alumnos. Claro está que la competitividad del centro escolar es aspecto fundamental para su supervivencia.

Han surgido varios modelos que buscan asegurar la calidad ofrecida por los centros escolares. A pesar de sus peculiaridades, se reconoce que un buen modelo de calidad educativa deberá basarse en la reflexión-acción del trabajo cotidiano para, posteriormente, aplicarlo a los conceptos que conforman una tríada fundamental de calidad: evaluación-acreditación-certificación.

Para entender la cultura de calidad, hoy tan difundida en el ámbito mundial, es necesario tener claros los conceptos de la tríada de calidad. A continuación se explicarán sus distintas acepciones.

\section{DEFINICIÓN DE TÉRMINOS}

En este trabajo, se entiende por evaluación el proceso de valoración que promueve la mejora mediante la detección de fortalezas y debilidades que ayuden a la toma de decisiones asertivas y al perfeccionamiento del propio proceso 
educativo. Dentro de este proceso de evaluación, se considera la acreditación como haber verificado el logro de los criterios y estándares de calidad previamente establecidos. Cabe aclarar que no es posible hacer referencia a la acreditación si no se lleva a cabo dentro del contexto de la evaluación. Finalizado este paso, se obtiene la certificación, que consiste en otorgar un reconocimiento por haber acreditado los distintos ámbitos de calidad que fueron evaluados por un organismo evaluador externo.

Hoy, las instituciones educativas se encuentran bajo la presión de demostrar su efectividad, por lo cual se han desarrollado distintos modelos a fin de evaluar si las instituciones educativas cumplen, o no, niveles de calidad.

Evaluación y calidad son dos términos clave que deben aclararse para participar en cualquier proyecto enfocado a la calidad. Por ello se explicarán ambos términos.

Es frecuente la confusión entre medición y evaluación. La medición consiste en asignar puntajes al rendimiento del estudiante: es simplemente un mecanismo útil para el proceso de evaluación educativa. Debe quedar claro que no es un fin en sí misma. Pero es la medición, la calificación, a lo que se reduce frecuentemente la evaluación, quedando así incompleto el proceso.

Pero más que la mera calificación, interesa la valoración del aprendizaje; es decir, la interpretación de esos puntajes para evaluar cómo va aprendiendo el estudiante. Lo cuantitativo es importante, pero sólo debe considerarse como un insumo de la evaluación; ésta involucra conceptualmente al término medición y tiene un significado de mayor amplitud (Kenneth, 2000: 22).

Es frecuente encontrar centros educativos que, buscando elevar los niveles de calidad, compran recursos novedosos, remodelan sus instalaciones, emplean herramientas tecnológicas, entre otras cosas. Esto puede coadyuvar, pero no es pieza clave de la calidad en los procesos y resultados educativos. En 
pocas palabras, cantidad no implica necesariamente calidad; calidad significa excelencia, ser integralmente mejor.

Ahora bien, evaluar la calidad de la educación no debe limitarse, como hasta hoy, al campo de los conocimientos, sino que necesariamente debe comprender, por igual, estos tres campos: conocimientos, procedimientos y actitudes (Filmus, 1999: 79). También podría agregarse el desarrollo de las aptitudes. Esto no olvida que la evaluación de las actitudes resulte mucho más compleja que la evaluación de conocimientos, por ello, la urgencia de ampliar la visión educativa de la evaluación a las demás áreas de desarrollo.

Esto es: se antepone la evaluación de la calidad como un mecanismo para medir los indicadores de calidad con la finalidad de lograr la mejora educativa.

"Entender lo que supone evaluar la calidad implica aceptar un significado para ambos términos que resulte coherente en un contexto cultural previamente definido. Los dos términos [evaluación y calidad] se prestan a múltiples interpretaciones y son, con frecuencia, utilizados de manera confusa para justificar, más que aclarar, los propios intereses o las argumentaciones técnicas de los agentes sociales" (Pérez Juste, 2001: 101).

En palabras de Schmelkes, la evaluación educativa es ahora una prioridad en América Latina. Los organismos internacionales de diverso tipo tienen diferencias entre ellos, incluso en sus políticas relativas a la evaluación educativa ${ }^{1 .}$ Asimismo, la evaluación educativa no causa la calidad de la educación, pero sin evaluación educativa no puede existir calidad de la educación (Ornelas, 2002: 163-164).

\footnotetext{
${ }^{1}$ Existen diversos tipos de organismos internacionales vinculados con la evaluación educativa y que tienen algo qué decir acerca de la evaluación realizada o a realizar en América Latina. Los tipos identificados son: a) los ligados a la banca multilateral (como el Banco Mundial); b) los intergubernamentales de apoyo mutuo (como la OCDE); c) los intergubernamentales de asistencia técnica (como la UNESCO y la OEI); y d) los no gubernamentales que agrupan centros de investigación (como la IEA).
} 
Dadas estas premisas, a continuación se explicará el concepto de calidad aplicado al ámbito educativo.

\section{EDUCACIÓN Y CALIDAD}

Hablar de calidad resulta complejo por sus diversas interpretaciones y definiciones; aún más difícil de definir, es el término de calidad educativa, por involucrar a dos términos en un mismo concepto. El término calidad proviene del latín qualitas que significa cualidad. "Este vocablo ha tenido diversas connotaciones, que podemos sintetizar en tres: tradicional, modernizante y dialéctica" (Kenneth, 2000: 32).

Por tanto, calidad se refiere a cualidad, comúnmente empleada como sinónimo de bueno, excelente; por eso, en caso de no existir calidad es necesario recurrir a un adjetivo o adverbio que la califique ("mala calidad", "poca calidad", "baja calidad"). Así, calidad en sentido absoluto se refiere a la excelencia del producto, del bien o del servicio; esto implica poseer las notas constitutivas propias de su ser (Pérez Juste, 2001: 22).

Ciertos autores consideran la calidad como un indicador meramente cuantitativo, como el caso de Kenneth al decir que: “(...) el vocablo cualidad sirve para designar metas, hábitos y capacidades que puedan someterse a medición objetiva. En consecuencia, se considera calidad de la educación a aquélla determinación de tendencia, cuya medición revele el grado en que los medios conducen de una manera más o menos rápida y directa hacia los objetivos" (Kenneth, 2000: 33). En esta definición se vislumbra una concepción de la evaluación como la mera acreditación; la calidad educativa debe ir más allá.

Al disertar sobre calidad de la educación, es frecuente identificarla con el rendimiento académico o de eficiencia externa o interna del sistema educativo, entendiendo así a la calidad como resultado y no como proceso educativo. 
Para alcanzar la calidad no es suficiente con realizar esfuerzos aislados, ni esfuerzos periódicos; es indispensable hacer extraordinariamente bien el trabajo diario. Esto es, alcanzar la excelencia al planear, realizar y evaluar las estrategias educativas cotidianas. Para ello es imprescindible definir cómo deben llevarse a cabo dichas estrategias.

Hablar de calidad educativa puede resultar un pleonasmo, pues "el concepto educación incluye en sí mismo la calidad. Se sabe que toda persona tiene derecho a la educación, pero éste no sólo es el derecho a una determinada cantidad de educación, sino el derecho a una determinada calidad" (García Hoz, 1982: 2). Por tanto, se concluye que ésta siempre debe poseer calidad; quienes manifiestan ofrecer alguna actividad relacionada con la educación que carezca de calidad, no están ejerciendo una verdadera educación.

La buena educación implica calidad. Según Ornelas, existen cuatro rasgos de una buena educación, "cada uno de ellos es una abstracción y por tanto una fragmentación de la realidad, porque en el plano de la experiencia humana todos son inseparables, están fundidos e integrados" (Ornelas, 2002: 42). Estos cuatro rasgos de la buena educación son: carácter, inteligencia, sentimientos y libertad; son cuatro esferas del desarrollo de la persona que se compenetran y fusionan.

Para entender y definir calidad educativa es necesaria la implicación de varios conceptos, ya que se trata de un término reciente que, sobre todo en el ámbito educativo, posee muchos significados o modos de empleo y también depende del concepto de educación que se posea. A continuación se presentan algunas definiciones presentadas por distintos autores:

Según Gastón Mialaret, la calidad "resulta del consenso existente entre las expectativas de la sociedad, expresadas en finalidades generales y objetivos particulares, por una parte y, 
por otra, de las características de los procesos en acción y las modificaciones operadas en los alumnos como consecuencia de tales procesos" (María de Allende, 1990: 250).

Por su parte, Velasco considera a la calidad como "una determinación del ser con relación a su perfección, se refiere al ser mismo de las cosas que, hablando de las personas, alude a sus caracteres psicológicos, espirituales" (Velasco, 2000: 33). Asimismo, afirma que "la educación de calidad es aquella que ayuda al educando a satisfacer plenamente sus necesidades y a desarrollar al máximo sus posibilidades personales de manera integral, contribuyendo así a su participación plena y constructiva en la sociedad en que vive de acuerdo a lo que ésta espera y necesita de él” (Velasco, 2000: 261).

La Organización para la Cooperación y el Desarrollo Económico, OCDE, en su documento de 1991, entiende calidad como atributo o conjunto de cualidades o características definitorias. El segundo sentido que da la OCDE es el de grado de excelencia o valor relativo: se le otorga aquí una interpretación normativa, e implica un juicio de valor y una posición en una escala implícita (o explícita) de bueno o malo (Velasco, 2000: 35); esto es, indica aquellos rasgos que son considerados como importantes dentro del proceso educativo.

Este organismo es el encargado de apoyar, económicamente, a países subdesarrollados para que puedan implementar las recomendaciones emitidas por la Organización de las Naciones Unidas para la Educación, la Ciencia y la Cultura (UNESCO).

La OCDE, en su documento de 2002, acerca del mejoramiento en la calidad del profesorado, menciona que una pieza clave para alcanzar la calidad educativa es elevar la calidad docente con miras a promover el desarrollo del aprendizaje de los alumnos. Considera que la calidad docente está basada en características observables y no observables (OCDE, 2002: 79-85): 
a) Características observables: Preparación académica del profesor y conocimiento acerca de la materia que imparte; estatus de certificación docente; evaluación de habilidades académicas; experiencia docente y tiempo, y grado obtenido en cuanto a su actividad profesional en la enseñanza.

b) Características no observables: Claridad y habilidad verbal; habilidades de comunicación en general; competencias de trabajo en equipo; competencias de trabajo en el aula; motivación para trabajar con los alumnos; seguimiento del progreso de los alumnos; flexibilidad; creatividad; tarea orientada al comportamiento; visión acerca de los propósitos de la educación. Cabe aclarar que las nombra como "no observables", porque implican una serie de evidencias que llevan al auditor a formar un criterio al respecto.

Esta visión sobre el papel preponderante del docente para alcanzar la calidad, es compartida por Sylvia Schmelkes, quien señala que el factor más importante de la calidad en la escuela es, sin duda, el docente (Schmelkes, 1997: 19). Reiteramos: el elemento más importante del centro educativo es el profesorado, pero este profesorado ha de trabajar en equipo, en el desarrollo de la capacidad de liderazgo de los docentes, no sólo de los directivos (Estruch, 1999: 9).

Así, Estruch concibe la calidad como la "mejora del servicio educativo en beneficio de los alumnos y de la sociedad" (Estruch, 1999: 137).

Siguiendo con algunas definiciones de calidad educativa, García Hoz afirma que podría describirse el término calidad educativa como el modo de ser de la educación, que reúne las características de integridad, coherencia y eficacia. En otras palabras: una educación tiene calidad en la media en que es completa, coherente y eficaz; la integridad incluye todos los factores necesarios para el desenvolvimiento del hombre; el 
orden y la coherencia se refieren al que han de tener cada uno de sus elementos, la importancia correspondiente a su papel en la vida humana, y asimismo su correlación; la eficiencia implica que todos los elementos cumplan adecuadamente su función (García Hoz, 1982: 3).

Como se observa, hay distintas definiciones -incluyentes pero no exclusivas- sobre el término; el problema radica en que esta confusión no ha permitido crear una definición válida para el término de calidad educativa. Ello puede deberse a tres factores (Pérez Juste, 2001: 15-17):

1. Reduccionismos en la definición.

2. Diversas concepciones de partida.

3. Modelos de referencia.

Algunos cuantos elementos constitutivos de calidad que definen los estudiosos son:

\begin{tabular}{|l|l|}
\hline AUTOR & ELEMENTOS CONSTITUTIVOS \\
\hline María de Allende & Principales factores: \\
(María de Allende, 1990: 250) & • El medio o entorno social. \\
& - El saber o las interpretaciones \\
& variadas de la calidad. \\
& - El educador. \\
& - El estudiante. \\
& - Los métodos y técnicas pedagógicas. \\
\hline Moreno & Valores fundamentales: \\
(Moreno, 2002: 114) & - La orientación al servicio. \\
& - El liderazgo. \\
& - La información. \\
& - La colaboración. \\
& - La comunicación. \\
& - La mejora continua. \\
\hline
\end{tabular}




\begin{tabular}{|c|c|}
\hline $\begin{array}{l}\text { Mañú } \\
\text { (Mañú, 1999: 114-115) }\end{array}$ & $\begin{array}{l}\text { Indicadores de calidad: } \\
\text { - El cumplimiento del proyecto educativo. } \\
\text { - Los resultados académicos. } \\
\text { - La satisfacción del personal. } \\
\text { - La matrícula para el curso próximo. } \\
\text { - Las circunstancias aclaratorias. }\end{array}$ \\
\hline $\begin{array}{l}\text { Pounder } \\
\text { (Pounder, 1999: 157) }\end{array}$ & $\begin{array}{l}\text { Dimensiones de efectividad: } \\
\text { - Productividad-eficiencia. } \\
\text { - Calidad. } \\
\text { - Cohesión. } \\
\text { - Adaptabilidad-a tiempo. } \\
\text { - Información sobre la comunicación } \\
\text { gerencial. } \\
\text { - Crecimiento. } \\
\text { - Establecimiento de objetivos planeados. } \\
\text { - Desarrollo de los recursos humanos. } \\
\text { - Estabilidad y control. }\end{array}$ \\
\hline Gestión de la Calidad Total & $\begin{array}{l}\text { Principios fundamentales: } \\
\text { - Orientación hacia el cliente. } \\
\text { - Mejora continua de los procesos. } \\
\text { - Autogestión. } \\
\text { - Autoevaluación. }\end{array}$ \\
\hline
\end{tabular}

México comienza ya el camino de la certificación de la calidad educativa, por lo que resulta interesante mirar a otros países, para conocer sus experiencias y aprendizajes. En Europa, se han desarrollado redes de apoyo para la calidad educativa, pero no son los únicos esfuerzos; a continuación se mencionan algunas de las muchas organizaciones dedicadas a la calidad educativa: 


\section{Perspectiva Internacional de los Modelos de Calidad Escolar:}

- Departamento de Educación y Ciencia de Gran Bretaña.

- Increasing Quality of Education for All (IQEA).

- Institute of Education de Cambridge.

- High Education Quality Council (HEQC).

- Comité Nacional d'Evaluation (CNE).

- Organización del Bachillerato Internacional (IBO).

- Agencia Nacional de Evaluación de la Calidad y Acreditación (ANECA).

- Total Quality Management (TQM). Programa enfocado a la calidad de la empresa.

- European Foundation for Quality Management (EFQM). Programa enfocado a la calidad de la empresa.

- International Organization for Standarization (ISO). Programa enfocado a la calidad de la industria.

- Council of International Schools (CIS).

\section{Perspectiva Nacional de}

\section{los Modelos de Calidad Escolar:}

a) Programa Nacional de Educación 2001-2006; menciona sólo algunos breves elementos cercanos a la calidad.

b) Programa Escuelas de Calidad (PEC) de la Secretaría de Educación Pública; es un programa público cuya misión es incorporar, en las escuelas públicas de educación básica, un nuevo modelo de gestión basado en los principios de liderazgo compartido, trabajo en equipo, respeto a la diferencia, planeación participativa, evaluación para la mejora continua, participación social responsable y rendición de cuentas.

c) Consejo de Normalización y Certificación de Competencia Laboral (CONOCER); su labor "gira en torno de la definición de normas técnicas de competencia laboral" (Argüelles, 1996: 48).

d) Premio Nacional de Calidad; tiene como misión promover 
y estimular la adopción de procesos integrales de calidad total con base en el Modelo Nacional para la Calidad Total, así como reconocer a las empresas industriales, comercializadoras y de servicios, instituciones educativas y dependencias de gobierno que operan en México, y que se distinguen por contar con las mejores prácticas de calidad total y mostrarlas como modelos a seguir por la comunidad mexicana.

e) Otros modelos.

Es frecuente que modelos surgidos en ámbitos concretos, por ejemplo la industria, se adopten sin la debida reflexión, análisis e implicaciones a otros campos, como puede ser el terreno de los servicios, tal es el caso de la educación. La dificultad principal que enfrentan los modelos de calidad empresarial, aplicados al ámbito educativo, es que no contemplan a la persona como el destinatario final de la calidad educativa, sino como un producto de calidad como cualquier otro.

Para mencionar un ejemplo de esta situación, se comenta la adaptación que ISO 9000 ha realizado para programas de certificación de educación superior. Como ya se ha señalado, el modelo de calidad de ISO 9000 inició para satisfacer estándares de calidad en la industria, con la intención de simplificar los modelos de producción; sus primeras acciones se llevaron a cabo a finales de los años 70 en la Gran Bretaña. Actualmente, existe muy poca bibliografía acerca de cómo se aplica ISO 9000 al ámbito educativo.

ISO, en su interés por el control estadístico, busca los resultados y, como medida de aseguramiento de la calidad, se enfoca a los procesos. Es posible obtener resultados, con una aparente calidad, que contengan procesos mediocres; por otro lado, si se verifican procesos de calidad, es más sencillo obtener resultados de calidad y, así, asegurar todo un círculo de la misma. 
Dicho modelo se basa en cuatro pasos que conforman el modelo de calidad: manual de procedimientos; récord de calidad; auditorías de calidad; y acciones correctivas al proceso. En específico, esta metodología busca mejorar las competencias y el desarrollo integral del estudiante, como resultado de las experiencias de aprendizaje. Considera a los estudiantes que ingresan como su "materia prima"; a los estudiantes que egresan como sus "clientes"; a la enseñanza basada en los programas como el "proceso" y a la evaluación basada en el plan de estudios como el "control de calidad" (Shutler, 1998: 152-161).

Para alcanzar cualquier certificación de calidad educativa es necesario que se involucren ante todo los directores, sin olvidar la necesaria participación de profesores, personal administrativo, alumnos y padres de familia; todos ellos, trabajando coordinadamente, estarán envueltos en el proceso de aseguramiento de la calidad interna.

Están en aumento los programas confeccionados a la medida de las necesidades de las organizaciones (Greensted, 1998: 142), por eso se plantea el diseño de un modelo de certificación, diseñado especialmente para adaptarse a las necesidades de cada institución educativa y que estandarice criterios comunes a ellas.

A continuación se presentan ideas generales que sustentan el proyecto personalizado de calidad:

La certificación sirve para apoyar el mejoramiento continuo de los centros educativos, y para esto, la certificación de un centro educativo implica, necesariamente, avalar el método de enseñanza-aprendizaje empleado por dicha organización.

Los objetivos del programa de Certificación de la Calidad en Centros Educativos son:

a. Promover el proceso de evaluación que favorezca la calidad y mejora continua de los centros educativos. 
b. Valorar a la evaluación como un elemento indispensable para la excelencia del centro escolar y, por consiguiente, de la sociedad.

c. Verificar el cumplimiento de procesos educativos de calidad que faciliten el alcanzar resultados en el aprendizaje de los alumnos.

Como evaluadores externos, se busca certificar a centros educativos de excelencia académica mediante un proceso de evaluación periódico que asegure - a la escuela y a la comunidad- que el centro cubre continuamente con altos estándares de calidad, con respecto a los conocimientos, habilidades y actitudes de alumnos, profesores y directivos; pues todo ello es reflejo de un proceso educativo de calidad.

Nos esforzamos por mejorar la formación de los educandos con la finalidad de hacerlos más libres, autónomos, mejores ciudadanos y mejores personas, por ello es vital la base antropológica del modelo. La finalidad de la calidad educativa es la mejora de la persona, para quien está dirigida la calidad.

Padres de familia, alumnos y comunidad, necesitan y merecen centros educativos de calidad. "Un centro que anteponga a los criterios pedagógicos la satisfacción de los clientes no está ofreciendo calidad educativa, sino mercadotecnia" (Estruch, 1999: 133).

Los mal nombrados "clientes" son, en primer lugar, los alumnos, los padres de familia, las agrupaciones sociales que apoyan al centro educativo y, en general, el Estado (Nagel, 1997: 101-102).

Para obtener la certificación, es necesaria la acreditación de estándares; los procesos de acreditación han sido reconocidos, en el ámbito educativo, como un método eficaz para iniciar y mantener la mejora continua del centro, a la vez que se apega a las regulaciones impuestas por el Estado. 
El punto de partida será la norma de calidad, considerada como el deber ser de los estándares y características de un bien o servicio.

Desprendidos de la norma están los criterios de certificación para los centros educativos que, al mismo tiempo, ayudan a asegurar tanto la calidad como la autonomía de la institución educativa:

1) Criterios personalizados: cada escuela requiere tener una filosofía organizacional y objetivos claros; mismos que deberán corresponderse.

2) Criterios estandarizados: cada escuela deberá cumplir con algunos elementos básicos para llevar a cabo su metodología. Antes de iniciar el proceso de certificación, es recomendable que los miembros del comité de calidad de la institución que desea certificarse, los conozcan y estén de acuerdo con ellos. A grandes rasgos, se refieren a contemplar, dentro de su modelo metodológico, los momentos didácticos (diagnóstico, planeación educativa, realización y evaluación educativa) y los elementos didácticos (actores del proceso enseñanza-aprendizaje, objetivos educativos, contenidos educativos, estrategias ${ }^{2}$, técnicas y procedimientos ${ }^{3}$, recursos didácticos, tiempo didáctico y lugar).

La clasificación de dichos criterios permite que el proceso educativo se consolide en los aspectos permanentes y se adapte a las circunstancias cambiantes.

Para llevar a cabo la verificación del cumplimiento de los criterios de certificación, se hará una revisión de estándares

\footnotetext{
${ }^{2}$ Entendidas como la metodología.

${ }^{3}$ Se entiende técnica como la táctica y procedimiento como el estilo docente y el estilo discente.
} 
de calidad que vaya de lo genérico a lo específico; además de estar en función de indicadores de calidad: cualitativos y cuantitativos considerados como condicionantes para la efectividad de una acción educativa.

Con la finalidad de asegurar los procesos de evaluación y acreditación, existe el Comité de Evaluación y Certificación, el cual estará conformado por representantes expertos de cada ámbito de certificación y por un Presidente. El voto de cada uno de ellos tendrá el valor de una unidad, constituyendo así un total de nueve.

Las evaluaciones e informes serán efectuados por el Comité de Evaluación y Certificación; los miembros del Comité deberán, en cada sesión, participar en el estudio y análisis de las solicitudes y reportes.

\section{MODELO DE CERTIFICACIÓN}

DE CENTROS EDUCATIVOS

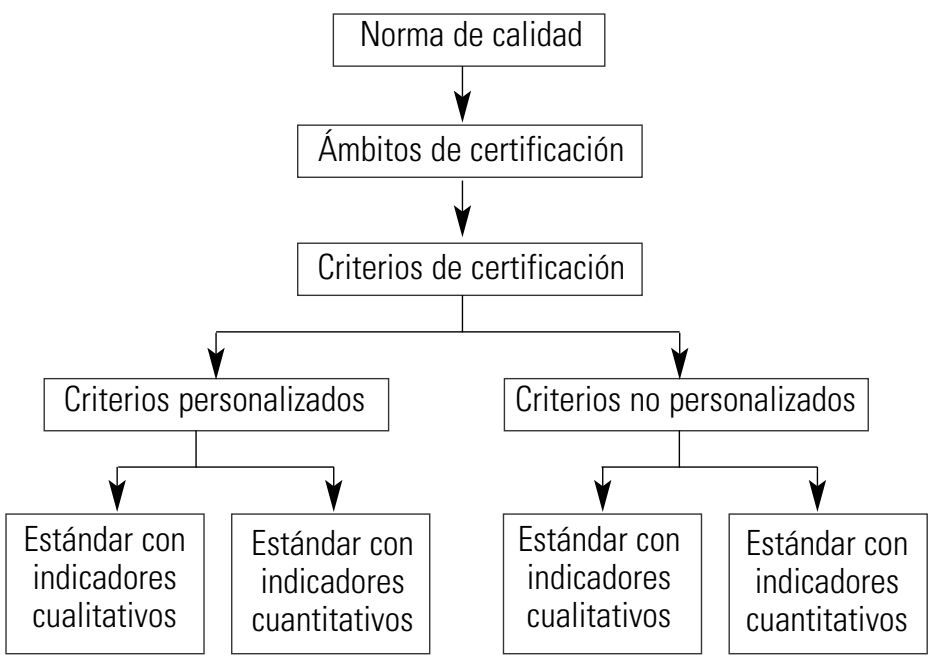


Existe la posibilidad de certificar todo el centro educativo o sólo algunas de sus áreas. La certificación está compuesta por los siguientes ámbitos de certificación $n^{4}$ :

\section{Finalidades, metas y objetivos:}

1.1. Filosofía organizacional y objetivos.

\section{Organización administrativa:}

2.1. Gobierno y administración del centro.

2.2. Funcionamiento administrativo.

\section{Estructura pedagógica:}

3.1. Modelo de enseñanza-aprendizaje.

\section{Contenidos educativos:}

4.1. Currículo del centro.

4.2. Recursos.

\section{Metodología, técnicas y procedimientos:}

5.1. Atención personalizada.

\section{Actores de proceso enseñanza-aprendizaje:}

6.1. Actividad docente.

6.2. Comunidad estudiantil.

\section{Resultados:}

7.1. Resultados en los alumnos.

7.2. Resultados en los ex alumnos.

7.3. Resultados en la sociedad.

\section{Escuela para padres:}

8.1. Estructura de la Escuela para Padres.

8.2. Funcionamiento de la Escuela para Padres.

${ }^{4}$ Elaborado con base en: VILLALOBOS PÉREZ-CORTES, Marveya., Educación comparada., UP-Ed. Cruz., 2002., p.p.129-133; y apoyado en las secciones y estándares del Council of Internacional Schools Accreditation Process, 2003. 
Para desarrollar el proceso de certificación, en cada una de estas áreas, se llevan a cabo los siguientes procedimientos:

I. Solicitud inicial.

II. Auto-estudio.

III. Solicitud de documentación.

IV. Visita preliminar.

V. Recomendaciones.

VI. Auditoría de evaluación.

VII. Acreditación.

VIII. Certificación.

IX. Reportes periódicos.

X. Re-certificación.

La solicitud inicial se concreta en un formato que solicita información al centro educativo, como es: nombre del centro educativo; datos particulares (dirección, teléfono, fax, entre otras); nombre y firma del director general; número total de la población; población por secciones; fecha de fundación del colegio; número de profesores que participarán en el proceso de certificación; y áreas del centro escolar que se desea evaluar. Se incluyen, igualmente, las razones por las cuales se pretende buscar la certificación.

Esta información es indispensable antes de comenzar el proyecto de certificación escolar; posteriormente, se realizará una visita inicial para conocer las instalaciones de la institución educativa y su funcionamiento; asimismo, se explicará al comité de calidad el procedimiento de trabajo para llevar a cabo el auto-estudio.

En esta misma reunión, se discutirán las cláusulas de calidad con el mismo comité para, posteriormente, elaborar una presentación del proyecto ante los profesores y personal administrativo. En caso de que la dirección lo considere pertinente, podrá hacerse una presentación a los padres de familia y a los alumnos. 
Posteriormente, continúa el auto-estudio que es la parte más importante de todo el proceso de evaluación y acreditación; se trata de un momento de evaluación interna e implica un serio compromiso por el tiempo y el esfuerzo que conlleva, no sólo para el líder de calidad, sino para todos y cada uno de los miembros de la comunidad educativa. El valor de los resultados de este auto-estudio es invaluable para la mejora del centro educativo. Generalmente, puede implicar el trabajo de un año escolar.

Dicha fase está compuesta por tres actividades principales: resolución del cuestionario; revisión de la filosofía y los objetivos; así como la autoevaluación de cada área del centro educativo.

Cada de uno de los ocho ámbitos deberán analizarse y describirse a la luz de sus objetivos, filosofía organizacional y los estándares de calidad. Al término, se pedirán conclusiones generales de cada apartado.

El auto-estudio debe basarse en la honestidad, en una visión integral del centro educativo y no deberá representar los intereses de un grupo, sino de toda la institución. Se recomienda repartir las actividades del auto-estudio entre todos y cada uno de los miembros del centro educativo.

Una vez entregado y analizado el auto-estudio, la instancia certificadora hará una solicitud de los documentos que considere pertinentes para cotejar información y resultados. Podrán solicitarse documentos correspondientes a cada ámbito de la certificación. La documentación será solicitada al Comité de Calidad del centro educativo y deberá entregarse en el período de tiempo acordado.

Como siguiente etapa, aparece la visita preliminar; durante ella, el centro educativo será visitado por un equipo de evaluadores cualificados en la docencia y administración de los mismos. Su visita se basará en las conclusiones entregadas en el auto-estudio, sin olvidar que el proceso de certificación estará basado en la filosofía y objetivos del centro; la visita implicará aproximadamente una semana y los visitantes entre- 
vistarán a los alumnos, profesores, directivos, personal administrativo y padres de familia. Los visitantes no tendrán el presupuesto de algún otro modelo escolar.

Los estándares se adaptan para asegurar su adecuación al funcionamiento del centro y cubrir los niveles de calidad mínimos requeridos.

Durante la visita, los miembros del equipo no darán a conocer ningún tipo de juicio valorativo; éste se entregará, posteriormente, a los miembros del Comité de Calidad. El equipo efectuará una valoración de la calidad educativa que ofrece el centro, no de la calidad profesional de los individuos que en él laboran.

Terminada la visita, el equipo se reunirá para elaborar un reporte detallado que abarque cada ámbito de certificación. Se compondrá por tres secciones: observaciones generales; recomendaciones y comparación con los estándares.

Con esta información, la institución certificadora emitirá su resolución en cuatro sentidos:

1.- Proceder a la certificación de toda la escuela.

2.- Proceder a la certificación de alguno de los ámbitos de calidad que cumplan con sus estándares.

3.- Realizar las recomendaciones para que, con base en las medidas correctivas, se alcance la acreditación de los ámbitos de certificación.

4.- Negar la certificación por estar muy distante de los estándares de calidad o haber incurrido en alguna falta ética en la elaboración del reporte del auto-estudio.

La recomendación es una sugerencia de cambio - no una imposición- para la mejora de la institución en la medida de sus posibilidades.

A continuación, un grupo de evaluadores externos hará la visita de auditoría en la que evaluarán cada uno de los 
estándares del o los ámbitos de certificación del centro escolar; los auditores deben ser expertos en la materia a evaluar.

Como parte de este proceso se harán, como máximo, dos visitas de auditoría; en el caso de acreditar los estándares en la primera visita, se procederá a la certificación; en caso contrario, se harán recomendaciones y se realizará una segunda y última visita de auditoría. En el caso de no acreditar, posteriormente a la segunda visita, se podrá acceder a una tercera, en el plazo que el organismo certificador considere pertinente.

Los resultados de las auditorías se darán a conocer en el plazo acordado, previo análisis y autorización del Consejo; dichos resultados podrán ser: acreditación o, en su defecto, nuevamente recomendaciones.

La finalidad de la auditoría será verificar si se han alcanzado los estándares de calidad para obtener la acreditación y/o hacer las recomendaciones pertinentes, a través del Comité de Calidad del centro educativo.

Se acredita a una institución una vez que se ha verificado el logro de los criterios y estándares de calidad previamente establecidos. En este caso, la siguiente etapa será la certificación derivada de haber acreditado uno o varios de los ámbitos de certificación.

Se entregarán dos categorías de certificados:

a) Certificado del centro escolar. Se otorgará a la escuela un reconocimiento que avale los ámbitos acreditados.

b) Certificado del docente: Tendrá derecho a obtenerlo, aquel docente evaluado que haya acreditado los estándares de calidad.

La validez de la certificación, en cualquiera de ambos casos, tendrá una vigencia de cinco años, con opción a re-certificarse terminado este período. 
Se pedirá un reporte anual de actividades ${ }^{5}$, donde se detalle el funcionamiento de las actividades correspondientes a cada ámbito de certificación.

Dicho reporte deberá entregarse en un período de entre once y trece meses ulteriores a la fecha de certificación y, posteriormente, de forma anual. En caso de que la instancia certificadora no reciba los mencionados reportes anuales, tendrá la facultad de retirar la certificación al centro escolar.

La re-certificación deberá estar presidida por los reportes periódicos, aunados a una nueva auditoría y análisis de la documentación solicitada al centro escolar. A pesar de existir ya un camino andado, se realizarán los procedimientos previos para alcanzar la certificación, empezando por el auto-estudio. Esta última etapa es sumamente necesaria dentro del modelo, para asegurar que se siguen acreditando los criterios de calidad en los ámbitos de certificación.

Se recomienda comenzar con el auto-estudio dentro del cuarto año posterior a la obtención de la certificación para, al quinto año, realizar las auditorías pertinentes y, al finalizarlo, recibir de nueva cuenta la certificación correspondiente.

En los años correspondientes al auto-estudio y a la re-certificación no será necesario entregar el informe anual de certificación correspondiente al centro escolar, pero sí se solicitarán los reportes de los docentes certificados.

Hasta aquí el proceso de certificación en cuanto tal; sin embargo, el centro educativo inmerso en el proceso de evaluación, en cualquiera de los ámbitos de certificación, queda comprometido al cumplimiento de un conjunto de deberes y, a la vez, amparado por una serie de derechos:

\footnotetext{
${ }^{5}$ Tanto del centro escolar certificado como de las actividades docentes de los profesores certificados y que aun colaboren en el mismo centro educativo en el que fueron evaluados.
} 


\section{Derechos:}

1. Referirse al proceso de evaluación y/o a la certificación recibida, en los documentos y formatos que emplee.

2. Disponer de las guías de auto-estudio y reportes de auditoría.

3. Disponer del asesoramiento técnico para llevar a cabo el auto-estudio, las recomendaciones de mejora y los reportes anuales.

4. Recibir la certificación correspondiente al haber acreditado el o los ámbitos de certificación evaluados.

5. La información recibida por la instancia certificadora o por personas involucradas en el proceso de evaluación institucional, es considerada como confidencial, salvo la correspondiente al informe final.

\section{Deberes:}

1. Realizar todas las fases, elaborar y entregar todos los documentos solicitados por la instancia certificadora; esto, dentro de los plazos y condiciones establecidas.

2. Notificar al organismo certificador, cualquier cambio que pueda afectar el proceso de certificación.

3. Permitir el libre acceso a sus instalaciones y colaborar con los evaluadores externos y demás personal en los procedimientos de auditoría.

4. Ejecutar, en la medida de sus posibilidades reales, los planes de mejora como consecuencia de las recomendaciones dadas.

Para concluir, podemos reafirmar que el centro educativo es considerado como una organización moderna formal, por ello la inminente necesidad de que tanto docentes como directivos estén ampliamente capacitados y posean las competencias necesarias para encaminar a la organización hacia la calidad. El centro educativo es, a su vez, una organización de servicios con un sistema abierto que le permite estar en continua interacción con el exterior; por ello, 
requiere estar al día en las exigencias que el entorno social va requiriendo para el avance social.

La propuesta de modelo de calidad de este documento, incluye la revisión del proyecto educativo y la influencia de la filosofía organizacional en todos los procesos que se llevan a cabo dentro del centro educativo. Para asegurar que el proyecto educativo se lleve a la práctica es recomendable realizar una evaluación institucional, que además permitirá revisar qué modificaciones se requieren para la mejora del mismo. La necesidad de la educación no se satisface a menos que la educación tenga la calidad necesaria.

La calidad educativa se traduce en hacer bien las cosas, como lo plantea el "Sistema de la obra bien hecha", de Víctor García Hoz. La calidad de la educación se manifiesta en la integridad de su planteamiento, en la coherencia de su realización y en la eficacia de sus resultados.

Para finalizar, cabe recordar que la certificación se basa en la calidad y la calidad es hacer bien las actividades de cada día en el centro escolar, buscando la mejora permanente en el alumno, el profesorado, las familias y la sociedad en la que está inmersa. 


\section{GLOSARIO DE TÉRMINOS}

Acreditación: La verificación en el logro de los criterios y estándares de calidad previamente establecidos.

Ámbito de certificación: Espacio de la organización educativa, dentro del cual se llevan a cabo una serie de procedimientos comunes para alcanzar un objetivo específico.

Auto-estudio: Proceso de evaluación interna; requisito previo a todo proceso de certificación y re-certificación.

Calidad: Grado de excelencia que alcanzan los procesos y resultados de un bien o servicio.

Certificación: Otorgar un reconocimiento por haber acreditado los distintos ámbitos de calidad que fueron evaluados por un organismo evaluador externo.

Criterio de certificación: Lineamientos de calidad basados en la norma y que pueden ser personalizados o no personalizados.

Cultura de calidad: Es la reflexión/acción de la organización, basada en la evaluación, acreditación y certificación.

Estándar de calidad: Nivel de calidad mínimo requerido en una actividad concreta.

Evaluación: Proceso de valoración que promueve la mejora mediante la detección de fortalezas y debilidades que ayuden a la toma de decisiones asertivas y al perfeccionamiento del propio proceso educativo.

Informe: Reporte elaborado con base en un auto-estudio o en una visita de auditoría; deberá contemplar el o los ámbitos de certificación.

Norma: Deber ser de los estándares y características de un bien o servicio.

Recomendación: Sugerencia de cambio para la mejora de la institución, en la medida de sus posibilidades. 
AINSCOW, M.; HOPKINS, D., SOUTWORTH; G. y WEST, M. (2001)., Hacia escuelas eficaces para todos: manual para la formación de equipos docentes., Madrid., Narcea.

ARGÜELLES, A. (1996)., Competencia laboral y educación basada en normas de competencia., México., Limusa.

ESTRUCH, J. (1999)., Dirección profesional y calidad educativa., España., Monografías Escuela Española.

FILMUS, D. (1999)., Los condicionantes de la calidad educativa., México., Novedades Educativas.

GARCÍA HOZ, V. (1982)., Calidad de educación, trabajo y libertad., Madrid., Dossat.

ISAACS, D. (1997)., Teoría y práctica de la dirección de los centros educativos., Pamplona., EUNSA.

KENNETH (2000)., Evaluación y calidad de la educación., Colombia., Magisterio.

MANÚ, J. M. (1999), Equipos directivos para centros educativos de calidad., Madrid., Rialp.

MARÍA DE ALLENDE, C. (1990)., Evaluación educativa: calidad de la educación., México., ANUIES.

MORENO, F. (2002)., Total quality and strategic planning in universities., Pamplona., EUNSA.

OECD (2002)., Teacher demand and supply: improving teaching quality and addressing teacher shortages., EDU/WKP (2002) 1.

ORNELAS, C. (2002)., Valores, calidad y educación., México., Santillana.

PÉREZ, J., LÓPEZ; F., PERALTA; M. y MUNICO, P. (2001)., Hacia una educación de calidad: gestión, instrumentos y evaluación., Madrid., Narcea.

SCHMELKES, S. (1997)., La calidad de la educación primaria., México., FCE.

VELASCO, J. L. (2000)., La participación de los profesores en la gestión de calidad de la educación., Pamplona., EUNSA. 
COMPSTON, Ch. (1998)., "Teacher preparation and certification: who, what, why and how"., en The History Teacher., Vol. 31., Issue. 4., p.519-531., JSTOR.

CHEONG CHENG, Y. y W. MING CHEUNG (1995)., "A framework for the analysis on educational policies"., en Quality Assurance in Education., Vol. 6., Num. 3., pp.141-144., MCB University Press., ISSN 0968-4883.

GREENSTED, Ch. y SLACK, J. (1998)., "Response to the QAA consultation paper on the quality assurance and standards framework for UK higher education"., en International Journal of Educational Management., Vol. 6., Num. 3., pp.141-144., MCB University Press., ISSN 0968-4883.

JAGADEESH, R. (2000)., "Assuring quality in management education: the Indian context"., en Quality Assurance in Education., Vol. 9., Num. 6., pp.10-21., MCB University Press Limited., ISSN 0951-354X.

LLOYD, S. (1991)., "National certification: a challenge to history teachers"., en The History Teacher., Vol. 24., Issue. 3., pp.313-319., JSTOR.

NAGEL, T. (1997)., "A dialogue about the quality of education"., Quality Assurance in Education., Vol. 5., Num. 2., pp.101-109., MCB University Press., ISSN 0968-4883.

POUNDER, J. (1999)., "Institutional performance in higher education: is quality a relevant concept?»., en Quality Assurance in Education., Vol. 7., Num. 3., pp.156-163., MCB University Press., ISSN 0968-4883.

SHUTLER, P. y CRAWFORD, L. (1998)., "The challenge of ISO 9000 certification in higher education"., en Quality Assurance in Education., Vol. 6., Num. 3., pp.152-161., MCB University Press., ISSN 0968-4883.

WAYNE, D. y WEIBLE, T. (1984)., «Teacher certification in fifty states: history/social science component", en The History Teacher., Vol. 17., Issue. 4., pp.537-543., JSTOR. 